\title{
THE STATE OF THE BRAIN DURING HYPNOSIS.
}

BY WILLIAM MaDOUGALT, M.A., M.B.

THE attempt to formulate a theory of the hypnotic state has been approached by two very different roads. On the one hand there is the road along which the late F. W. H. Myers so brilliantly led the attack. Those who follow this road fix their attention on the most strange and perplexing of the phenomena of hypnotism; they seek to exhibit the gulf between the normal and the hypnotic states as very wide; they incline to regard the hypnotic condition as essentially one of extended faculty and increased mental power; and they value hypnotic experiment as one way of approaching the study of alleged supernormal and marvellous phenomena, belief in which orthodox science regards as a delusion bred by erroneous observation and engendered by an incorrigible yearning after empirical evidence of a realm of purely psychical existence.

The other more sober way in which the task of explaining hypnotic phenomena may be approached is to concentrate attention upon the simplest and least astonishing of them, to seek out and establish the affinities of hypnosis with better-known mental states, and to try to find some hypothesis that will account for these simpler facts and will bring our knowledge of hypnosis into line with the facts of our normal mental life and with theories that have proved their value in other departments of research; for, if any such working hypothesis can be formulated, it may be hoped that its field of application may be gradually enlarged and that the more perplexing phenomena may be brought within its range as our knowledge of them increases.

Though I would not disparage or undervalue speculations of the former kind, I believe that the second way of approach is more consistent with scientific principles, and the following pages present the results of my own gropings along this road.

The aim of this paper is to render a little clearer and more definite in detail a hypothesis which has been gradually taking shape during recent years under the hands of a number of writers, and which goes by 
the name of the theory of cerebral dissociation; for certain unorthodox views as to the nature of cerebral processes set out by me in former numbers of this Journal [6] and elsewhere seem to lend themselves very well to this purpose.

The current loose usage of the phrase "theory of dissociation" tends to the confusion of several different hypotheses which have little. in common with one another, and some of which are held by authors who follow the former of the two roads distinguished above. There is the doctrine (or group of allied doctrines) which teaches that each normal personality comprises two selves, two personalities or strata of personality, which normally are in some sense fused together-the waking and the subwaking selves of Boris Sidis [9], the Doppel-Ich of Max Dessoir [2], the waking consciousness and the dream-consciousr.sss, the primary and secondary personalities, of other authors ${ }^{1}$; and that mental dissociation somehow consists in the freeing of the lower, or normally submerged and hidden, stratum from the dominance and control of the upper stratum. I. do not think that at the present time we are compelled to make, or are justified in making, the assumption that the normal human mind comprises any such submerged and coherent system of faculties as is implied by each and all of these allied doctrines.

More nearly allied to the form of the theory of dissociation that - I am concerned to support is Pierre Janet's doctrine of mental disaggregation [4]. Janet speculates only in terms of psychical material, and refuses to attempt a corresponding neurological hypothesis; but $I$ am very decidedly of the opinion that here, if anywhere, attempts at purely psychological explanation are out of place. I believe that any explanation 'or theory of the hypnotic state and phenomena must be psycho-physiological; for it seems clear that, during hypnosis, the brain is in some abnormal condition and functions in some manner different from that of the normal waking state, and that theiefore we cannot claim to understand hypnosis until we learn the nature of this abnormal brain state; that, in short, a description of the abnormal state of the brain must be an essential, if not the essential, feature of any explanation of the facts.

Th. Lipps [5], of Munich, also has proposed an explanation which, although he states it in terms of psychical dispositions only, and so limits its usefulness, seems to me very helpful ; and it can easily be translated into terms of psycho-physical dispositions and developed on those lines.

\footnotetext{
1 Possibly the doctrine of the Sublimal Self, elaborated by the late F. W. H. Myers, belongs to this group of theories, but I do not feel myself competent to class it.
} 
Lastly, I would mention the speculations of Oscar Vogt [3] (accepted and endorsed by Forel) as those with which my own views, although independently reached, have the closest affinity.

It is held by many authors, notably by Bernheim-rightly, I think-that hypnosis is a state closely allied in many respects to normal sleep, although it presents important differences. The onset of the two states is very similar in many (if not all) cases, and it is favoured in both cases by the same influences and conditions, namely, by the withdrawal of all strong sense-stimuli, by restful position, by monotonous gentle stimulation of one or more of the senses, by expectation and habit, and by the banishment of exciting thoughts and the concentration of attention on some unexciting object or sense-impression.

Another point of similarity of the onset of the two states is the heaviness of the eyelids, which is generally the first symptom of both sleep and hypnosis, and which, in both cases, is generally acconlpanied by a general drowsiness and disinclination to make any effort or movement. ${ }^{1}$

In both states the subject lies inert and passive; his mind is less responsive than during the waking state to most of the sense-impressions from the outer world; and, although it is not completely shut off from their influence, it is apt to interpret them falsely. In both states the subject may entertain the most fantastic ideas, ideas absolutely incompatible with his actual situation or his best-established convictions, without recognizing their absurdity; and such ideas are apt to undergo some fantastic elaboration in keeping with any prevalent emotion; in both cases these ideational processes are characterized by a lack of the voluntary and critical control which is habitual and normal in the waking state. In both cases the subject not infrequently desires to move his limbs, but finds himself incapable of doing so. In both cases, again, the subject is very apt to have, when awakened, no spontaneous recollection of the ideas of the period (of sleep or hypnosis), though recollection can generally be evoked by

I I am, of course, aware that some subjects exhibit while in hypnosis a degree of liveliness and excitability apparently greater than the normal, and that some of their faculties nuay seem even more alert and active than during their normal waking life. But these are dis. tiuctly exceptional cases, and usually, I think, this lively state is ouly arrived at after some trainiug. We have to concentrate our attention on the more usual type of hypnosis; for the symptoms of the state are so bewilderingly diverse and numerous that, if we attempt to form a theory which shall account at once equally well for all of them, it is obvious we shall fail. Very much depends upon the handling of each case by the operator. In my own experience I have generally aimed at inducing a sleep-like condition, and have always obtained this result, except where I have failed to induce any degree of hypnosis. 
appropriate questioning. Some subjects may be induced to display an abnormal suggestibility during normal sleep, and in some the cataleptic plasticity of the limbs, which is a so frequent symptom of hypnosis, may be induced during normal sleep.

Lastly, if the subject in hypnosis is left to himself, he is apt to pass spontaneously into normal sleep, the transition from the one state to the other being perfectly gradual, so that no line can be drawn between them ; and, conversely, the state of normal sleep may be converted into hypnosis.

The resemblance between hypnosis and sleep is, in some respects, still closer in the case of ordinary sleep-walking or somnambulism, which may perhaps be regarded as a state intermediate in character to these two.

On the other hand, hypnosis differs from normal sleep in that it has certain positive characteristics that are not found in the latter. A large proportion of these may be summed up by saying that the subject remains peculiarly sensitive to the influence of the hypnotizer, and that ideas introduced to his mind by the hypnotizer operate with unusual energy and effectiveness, or, as we say, the subject in hypnosis is very suggestible towards the operator, or readily takes suggestions from him.

The obvious line of approach is, then, to find a good working hypothesis as to the state of the brain in normal sleep; and, having formulated this, to try to understand what further peculiarities of the state of the brain are connected with the positive features in which hypnosis differs from sleep.

Unfortunately we have as yet no generally accepted theory of sleep. We have a number: of partial theories or views-the view that sleep is due to the action of waste products of metabolism on the nervous tissue; the view that it is due to cerebral anæmia; the view that it is due to auto-suggestion; the recent view of Claparède [1] that the onset of sleep is an instinctive process.

There is probably some truth in all of these views, as well as in others; but neither individually nor collectively do they give an answer to the essential question: How does the state of the nervous tissue of the brain during sleep differ from its state during waking life?

It seems clear that during sleep the nervous substance of the cerebral hemispheres is either less excitable than during waking life or. transmits any excitement less readily from part to part; and in all probability both these statements are true. A high degree of excitability and great freedom of transmission of excitement between all parts - these seem to be the essential positive characters of the waking brain 
that are lacking to the sleeping brain. Our definition of the state of the brain during sleep must, then, be a negative definition; it must consist in denying it the condition which underlies this proneness to activity characteristic of the waking state.

What, then, is this peculiarity of the waking state? The clue to the answer to this question is given, I think, by those rare cases of very widespread anæsthesia, of which the most striking and most frequently cited example is the case described by Strümpell [10]. The patient was a boy completely anæesthetic as regards all his sense-organs and sensory surfaces, with the exception of one eye and one ear. Whenever the sound eye was closed and the sound ear stopped, this patient fell almost immediately into deep sleep, and could then only be wakened by flashing a light into the sound eve or shouting into the sound ear, or otherwise stimulating violently one or both of these organs. That is to say, in this case the waking state was maintained only by the constant incidence of impressions on these sense-organs.

Now, it seems in the highest degree probable that the constant rain of stimuli upon our sense-organs plays a similar part in maintaining the waking state of the normal man; but the fact is obscured in two ways: firstly, by the great variety and sensitivity of the sense-organs of the normal man and the impossibility of shielding all of them from stimulalation; secondly, by what may be called the capacity of the human psycho-physical organism for maintaining an endogenous excitation. We must briefly consider these in turn.

Even if one lies still on a soft bed in a quiet dark place; the nervous system is yet liable to receive many stimuli, especially through the senseorgans of the kinæsthetic and visceral systems. Under these conditions we often become aware of these impressions, and often they excite some sense of discomfort, some need of movement or change, so that one may be kept turning restlessly without sleep. The most striking example of the power of these vague sense-impressions arising within the body to prevent sleep is afforded by those which excite the sensation of hunger.

Nevertheless, save in conditions of great fatigue, the withdrawal of stimuli from the principal sense-organs is a favourable and, for most of us, an essential condition of the onset of sleep. When sleep comes, its continuance depends upon the absence of strong sense-impressions; it may normally be terminated at any time by a sufficiently strong sensory stimulus, or by the summation of effects of a series of sensory stimuli, each of which is too weak to produce any marked change of state $;^{1}$ and

'I have described and discussed this process in some detail in the pages of $M i n d$ [7]. 
even after sleep is thus banished we do not usually feel ourselves to be fully awake and alert until a further stream of stimuli has impinged upon our sense-organs.

Now, unfortunately, our knowledge of the immediate effect of stimulation of a sensory nerve is still very imperfect; but there is much to be said for the view that the immediate and essential effect is a katabolic process which liberates chemically stored energy in the substance of the neurone, and that the spread of excitation consists in the discharge of this freed nervous energy from neurone to neurone across the synapses or places of junction of the neurones. ${ }^{1}$ This distinction between chemically stored or potential nervous energy and the liberated active nervous energy is, I feel sure, one of the first importance for neurological speculation, although but little attention is commonly paid to it. Oscar Vogt has recognized its importance and has proposed to mark it by calling the freed nervous energy "neurokyme." Some years ago I, in ignorance of Vogt's work, proposed to call it "neurin." What name we use does not much matter, so long as we hold fast to this distinction and to this conception of liberated active nervous energy; but for this purpose some name is essential; since Vogt's proposal was prior to my own, and since my proposed name is so similar to neurine, the name of one of the chemical compounds found by the chemists in nervous tissue, I adopt Vogt's term " neurokyme " in place of " neurin."

During waking life, then, stimuli rain unceasingly on all the senseorgans and liberate in all the sensory nerves streams of neurokyme, which ascend by the sensory tracts of the cord and lower brain to the cerebellum and cerebrum. The brain is thus fed and its activity Is sustained by these streams of energy, which keep it charged with

' Some arguments in support of this view are adduced in two papers in former numbers of this Journal [6]. In a recent paper, privately printed (Proceedings of the Jumior Scientific Society, Oxford), I have ventured to suggest that this way of conceiving the immediate effect of a stimulus upon a nervo affords a possible explanation of the fact that the amount of nervous response varies with the intensity of the stimulus applied. We may suppose that the katabolic change propagates itself along the substance of a neurone on the all-or-nothing principle, to which an explosivo decomposition may be expected to conform; but we may suppose that the quantity of such change in unit of time varies with the energy of the incident stimulus, as in the case of a train of damp gunpowder. The distance through which the change will propagate itself, or, in other words, the length of nerve fibre, and hence the amount of nervous substance which undergoes the katabolic change, will, then, vary with the intensity of the stimulus; and since the quantity of energy liberated will vary with the amount of nervous substance which undergoes this change, it also will vary with the intensity of the stimulus. A maximal stimulus will, thon, cause the katabolic process to propagate itself throughout the whole substance of the neurone, and every submaximal stimulus will cause it to propagate itself through a part only of tho neurone substance, the length of which part will be proportional to the intensity of the stimulus. I throw out this suggestion with the greatest diffidence for the consideration of those who are better qualified than myself to say whether it is a tenable hypothesis. 
neurokyme at a varying tension or potential; and this charge of free energy is constantly being worked off by thought or mental activity of any kind, for all mental activity involves the discharge of neurokyme from the sensory to the motor side of the brain, in accordance with James's law of forward conduction.

Now consider the second condition that obscures the importance of sense-stimuli for the maintenance of the waking state-what was called above the capacity for endogenous or automatic maintenance of the state of excitation.

The organism comprises certain hereditary pyscho-physical dispositions, which, in the evolutionary sense, are essentially continuous with, or to be identified with, the instincts of the animals. In what each such disposition exactly consists we do not yet know, though no doubt an essential feature of it is a complex system of sensori-motor arcs. In the present connexion the important fact is that each such disposition is a great spring of nervous energy; when anyone is excited in any way it liberates a great quantity of neurokyme that raises the activity of the brain to a higher level, a fact which manifests itself in symptoms of general excitement, in very energetic thought and action and, subjectively, in the form of impulse, desire, and emotion.

These dispositions can be excited by way of sense-presentation; hence sense-impressions contribute to the maintenance of the state of general excitation of the brain, not only in proportion to the intensity of the stimuli and the extent of sensory surface affected, but also in proportion as they lead to the excitement of any of these special springs of energy. In most of the animals these dispositions can be excited only by sense-impressions, but in the human being they can be excited also by way of representative or ideational processes. Hence the human mind and brain do not necessarily come to rest as soon as all sensory stimuli are withdrawn; the activity of an excitable brain may continue to be sustained by this process of endogenous liberation of energy, by the power of the impulses awakened through ideas and recollections. When this is the case, sleep can only be secured by the avoidance of emotionally exciting ideas, i.e., by turning the attention to indifferent things-to sheep jumping through a gap in a hedge, to counting, or to some faint bodily impression.

The presence in the brain-neurones of a store of free energy or neurokyme derived from these two sources is, then, a prime condition of the waking state; but there is a second important condition, dependent in large measure upon this one, which underlies the freedom of 
transmission of excitation from point to point of the brain, the free interplay of all parts of the brain, that is characteristic of the waking state; this is the state of the synapses.

We can confidently infer that the neurones that make up the nervous tissue of the cerebrum are connected together to form functional groups, the nembers of each group being so intimately connected that exciteruent of any one member of the group tends to spread at once throughout the group to every member of it.

Such a group of neurones is a functional unit, and we may call it a psycho-physical disposition; of such groups the hereditary dispositions mentioned above constitute a very important and peculiar class. We may infer also that these dispositions are connected with one another with various degrees of intimacy to form systems; these, again, with less degrees of intimacy to form larger systems; and these yet again with still less intimacy to form still larger systems; and so on, until we reach the most comprehensive system, which is the whole of the central nervous system.

Each disposition is an intricately woven chain of neurones making up a complex sensori-motor arc or system of arcs. All thought, all perceptual or ideational mental process, involves the perpetual shifting of the main nervous current from one disposition to another, at any one moment some one disposition being the main path of discharge of neurokyme from the sensory side of the brain, where it is constantly accumulating, to the motol: side; and, while any one disposition thus predominantly active is.the principal focus of excitation, those most intimately connected with it are in a state of subexcitement. When any one disposition thus becomes the main path of discharge, it is because, owing to a favourable conjunction of circumstances, it has become for the moment the path of least resistance from sensory to motor side of the brain. The discharge through any one disposition is the neural concomitant of the rise to consciousness of a corresponding presentation or idea; and the shifting of the main stream from one disposition to another is the neural concomitant of the play of ideas, of the succession of presentations at the focus of consciousness, which continues so long as we are awake.

An essential feature of the view I am expounding is that the various degrees of intimacy of connexion between neurones and between groups and systems of neurones are held to be functions of the synapses or junctions of neurones. If excitement spreads readily from one group to another, it is because the synapses on the path 
connecting those two groups present at that moment a low degrce of resistance; if it spreads less readily to another group, it is because the synapses on this connecting path present a greater resistance. Now there are many good reasons for believing that the resistance presented by any synapse is not a fixed quantity, and that it is not only permanently diminished in some degree by repeated transwission of the excitation-process, but that it is a quantity which varies from moment to moment under a number of infuences, of which the most important are fatigue of its own substance, chemical influences from the blood, and the charge or potential of charge of neurokyme in the neurones between which the synapse forms a junction. That is to say, it is maintained that each synapse (in the resting condition of the part) presents a certain normal degree of resistance which varies from synapse to synapse, and is in each case a fixed quantity (or one only slightly or slowly changeable) determined by heredity and the course of individual experience; but that this normal degree, to which the resistance of each synapse returns when the brain is at rest, is constantly liable to be modified by the influences named above, being raised by fatigue and anæsthetic drugs such as alcohol and chloroform, diminished by strychnine and tetanus-toxin and by the excitement of the neurones between which the synapse lies.

This last condition is the most important one in view of the problem in hand. I assume, and the assumption is not without positive evidence in its favour, that the resistance of the synapse falls as the potential of charge of neurokyme rises in both, or in either one, of the neurones between which it lies, and that it rises as this potential falls.

In the waking state, then, the hemispheres being constantly supplied with large quantities of neurokyme from the two sources indicated above, the main mass of its neurones is kept moderately charged with this free energy, the result of which is that all synapses, and therefore all connecting paths, are kept in a state of partially lowered resistance; and there is, therefore, a constant free interplay between all parts of the brain, the main current of energy shifting freely from one disposition to another and from one system to another, each disposition tending to draw to itself a maximal stream of energy, each competing with all the rest for the fullest share of energy according to the principle of drainage.

Now, when we lie down to sleep in a quiet dark place, we shut off as nearly as possible all stimuli from the sense-organs, and we divert our: thoughts from all emotionally exciting topics. The supply of neurokyme 
to the brain is thus diminished, the charge present in, or banked up in, the neurones of the afferent side of the brain falls to a lower potential, and, consequently, the resistance of the synapses in general rises. When sleep ensues from great general fatigue, another factor probably plays the principal part, namely, the waste products of metabolism, which, accumulated in the blood and lymph that bathe the synapses, act upon them, like chloroform or alcohol, as poisons which diminish their metabolism and so raise their resistance. Also, in the production of the sleep that ensues from deficient energy of the heart's action or from diminished circulation of blood in the brain, however produced, this second factor probably plays a large part, the waste products being allowed to accumulate locally. In normal falling asleep these two conditions-the general accumulation of waste products in the blood and the general slowing of the circulation-coöperate with the diminution of supply of neurokyme to raise the resistances of the synapses of all parts of the brain.

This general raising of the synaptic l'esistances throws the whole brain into a condition of relative dissociation or functional dissociation; that is to say, the dispositions and systems of dispositions, as well as the neurones comprised within any one disposition, become in some degree functionally isolated or separated from one another. And this functional discontinuity will be most complete in the case of the least intimately connected systems, less complete between the more intimately connected dispositions of any one system, and least between the neurones that are united in one disposition; for the resistance of each synapse will be reduced to, or near to, its normal resting degree.

Normal sleep implies, then, a state of relative dissociation of the brain, and the many points of similarity noted above between sleep and hypnosis indicate that hypnosis also involves relative dissociation of the brain; on the other hand, some of the phenomena of hypnosis, to bo noticed below, afford positive evidence that such dissociation obtains, and so confirm the indications afforded by the foregoing consideration of the general physiology of the brain and of sleep.

We have to inquire: How does the state of the brain during hypnosis differ from this state of general relative dissociation of normal sleep? The answer to this question suggests itself when we consider the way in which hypnosis is commonly induced. The onset of hypnosis is favoured by the influences which favour sleep (with the exception, possibly, of fatigue), namely rest and quiet (i.e., the withdrawal of sensory stimuli), the slowing of the circulation, the banishment of emotionally exciting 
thoughts, and by the expectation of sleep. How expectation operates remains a very obscure problem, but it is clear, I think, that in neither: case is it an essential factor. The important influences brought to bear in the induction of hypnosis, in addition to those which normally produce sleep, are: (1) monotonous stimulation of sense-organs, either continued (as by visual fixation of a bright point) or intermittent (as by passes); such monotonous stimulation is favourable also to the onset of normal sleep; (2) the personal contact of the hypnotizer, who, by speech, by verbal suggestions, and by manipulations, keeps the subject constantly aware of his presence.

The monotonous stimulation seems to aid in bringing the whole brain to a quiescent condition, by facilitating the continued direction of attention to an object or impression of an unexciting uninteresting character, and thereby preventing the free play of ideas which otherwise may maintain itself for a considerable period in the way noted above. In terms of neural process we may say that the monotonous stimulation tends to keep some one minor disposition or small system of dispositions in dominant activity, keeps open this one path of discharge, so that this one channel, constantly draining off from the sensory side of the brain the supply of neurokyme, depresses, or tends to prevent, the activity of all others. ${ }^{1}$

The personal contact of the operator contributes to produce the same result. His passes, his manipulations, his verbal suggestions, all serve to keep the idea of the operator present to the mind of the subject, to keep the subject's attention (no doubt an attention of low grade or potential) directed to the operator; i.e., in terms of neural process, they serve to keep in a state of excitation one system of psycho-physical dispositions, the system whose activity underlies the presence to consciousness of all thought of the operator ; or, again, they tend to keep the main current. of nervous energy shifting from one disposition to another within this one system. In this way, while all the rest of the brain is allowed to sink into a state of quiescence and of relative dissociation similar to that which obtains in normal sleep, this one system is kept active and waking, so to speak. It thus serves as an open channel through which ideas can be introduced to, or evoked in, the mind of the subject, as a single focus of nervous activity in a quiescent brain, from which focus other parts of

\footnotetext{
1 That inhibition within the nervous system is always and at all levels a process of drainage of energy from one path or system to another in virtue of the lower resistance presented by the inbibiting path is a view that, as I have tried to show in a previous paper (Brain, vol. xxvi.), is compatible with all the facts and seems to be the only tenable working hypothesis.
} 
the brain may be brought into play. Any proposition made by the operator to the subject is then accepted uncritically and acted upon because accepted with belief-that is the essence of suggestion-whereas the subject is blind and deaf to impressions from all other persons and objects, except in so far as they are connected in his mind with the operator, i.e., except in so far as they belong to the same system of ideas.

Now a leading feature of hypnosis is that ideas or propositions suggested by the hypnotizer not only are accepted, but, being accepted, operate with a quite unusual force or effectiveness in the mind and on the body of the subject. The state of the brain described above, the state of relative dissociation of all systems except the one, enables us to suggest an explanation of this feature also. In the normal waking state any proposition about any topic or object is received more or less critically, and is only accepted with conviction if it is not incompatible with the organized body of knowledge or belief about that topic or kind of object already established in the mind. Every idea, we may say, has to withstand or overcome the inhibiting tendencies of these other ideas connected with the same topic before it is fully accepted, before it can prevail stably and determine action in the way characteristic of belief. But in the state of relative dissociation, any idea introduced to the mind by the operator prevails stably and determines action-is, in fact, accepted with belief-just because the ideas which could check or weaken its operation are not aroused, are not brought to bear upon it in criticism, owing to the state of relative dissociation which renders all interplay of ideas more difficult, more sluggish, than in the waking state. Further, in the waking state not only contradictory ideas, but all ideas whatsoever that have any tendency to rise to consciousness at the moment, play a similar part, weakening to some extent the force with which the dominant idea at the focus of consciousness operates in the mind and on the body. ${ }^{1}$ The refined experimental researches of G. E. Müller upon reproduction and association seem to have established this fact [8].

We may try to express these relations in physiological terms. We must remember: that in the waking state of the brain all dispositions and systems of dispositions are in relation of reciprocal inhibition with one another, such that the activity of any one tends to inhibit the activity of every other; and we may fairly suppose that between dispositions whose activities underlie incompatible or contradictory ideas about any object,

\footnotetext{
'I am, of course, using the word idea to denote the whole psycho-physical system of activity which reveals itself in consciousness as a presentation.
}

BRMIN.-VoL. XXXI. 
this relation of reciprocal inhibition is peculiarly intimate and direct. ${ }^{1}$ All dispositions, then, compete with another, except those that form a harmonious system and tend to express themselves in some particular: mode of coördinated bodily activity. In the waking state, then, the energy with which any idea tends to express itself, or realize itself through bodily action, is thus diminished by the competition of all other ideas that have any tendency to rise to consciousness; or, in neural terms, the energy with which any disposition functions is normally to some extent depressed by the competition of all other dispositions that are in any state of subexcitation, and especially of those of contradictory ideas. In hypnosis, on the other hand, this depressing, weakening influence, this partial inhibition, is abolished or diminished in virtue of, and in proportion to the degree of, relative dissociation or functional isolation of dispositions from one another. Hence, any idea suggested by the hypnotizer is not only accepted uncritically, but operates with greater force than any idea accepted with conviction in the waking state.

The absence or diminution of all such inhibitory weakening and restraint, and the correlative concentration of all available neurokyme along the channels of one disposition, seem to be the principal factors to be taken into account when we seek to explain all the commonest and most easily produced results of hypnotic suggestion, namely, the illusions, positive hallucinations, ${ }^{2}$ delusions, the control of the voluntary

1 The bypothesis of inbibition by drainage seems to lend itself well to the explanation of this kind of inhibition, although, of course, it is not possible to apply it in detailed fashion. iVe may liken the relation between the dispositions of contradictory ideas to the relation (so brilliautly studied by Sherrington-see "Integrative Action of the Nervous System ") obtaining between the reflex arcs innervating antagonistic muscle.groups; and this case is not only truly analogous, but is probably more than analogous; it is probably the simplest example of the same type of functional relation. Contradictory ideas about an object tend to issue in opposed systems of muscular activity.

"It is perhaps worth while to expound the application of the hypothesis to one of these cases a little more in detail. Take the case of induction of a positive hallucination. It is suggested to a subject that a blank card carries a photograph of some fumiliar building. Perhaps the subject says he can see no picture on the card. A few irregular lines drawn on the card will then often facilitate the induction of the hallucination, and on repetition of the suggestion the subject may then admit that he sees the picture, and may describe it in some detail, and may even continue to see it on the same card when awakened, without postbypnotic suggestion to that effect having been given. The influence of the lines drawn on the card in facilitating the induction of the hallucination (which in this case approximates to the type of illusion rather than pure hallucination) is significant. There is good reason to believe that the lines, serving as points de repere, are worked up among the hallucinatory lines of the subject's picture. We may suppose that the verbal suggestion of the picture of the familiar object evoked, in the mind of the subject, an image or visual representation of the building, which, when he directs his gaze to the card, is projected upon the card with hallu. cinatory or sensory vividness. I have sometimes been able to follow this order of events-the formation of a visual representation of an object while the eyes are closed and the subsequent projection of this image, with increasing vividness and distinctness, when the subject is directed to open his eyes upon a card. The neural events we may conceive as follows: the rise to consciousness of the visual image results from the spread of excitation to the 
muscles and (to some extent) of the involuntary muscles and of the visceral processes, secretion, nutrition, and so forth. While, as Pierre Janet has suggested, cataleptic plasticity of the limbs may be equally well regarded as due to the functional isolation of the cerebral tracts by which the afferent impulses ascending from the organs of the " muscular sense" return to those motor elements of the cortex from which the same movements and positions of the same parts are voluntarily effected (in accordance with the principle of the upper motor circuits). The weakening or abolition of reflexes, which, I believe, occurs only in very deep stages of hypnosis, may be regarded as due to the dissociation having attained so great a degree as to affect the functional continuity of the neurones composing the reflex arc.

To negative hallucinations, and to the execution of post-hypnotic suggestions by a subject who remains unaware of the nature of the suggestions given, these principles of explanation are not so easily applicable; that is to say, while cerebral dissociation is implied by them, the principle is not in itself adequate to shadow forth an explanation of them; some further principle is implied. But these processes are especially interesting from the present point of view because they prove, more clearly than any other of the phenomena, that some functional dissociation of the brain is really present. In both cases we have unmistakable evidence that some process goes on in the brain independently of, and without affecting or being involved in, the main stream of psycho-physical process. Such, for example, is the deliberate ignoring by the subject of an object of which he has been told that it is no longer present, for although he certainly does not perceive the object

corresponding visual disposition from the auditory disposition directly excited by the verbal suggestion. If the subject were in the normal state and directed his gaze to the card, the lines upon it would be seen for what they are and the image would fade from consciousness as the attention was diverted to these lines; that is to say the disposition concerned in the production of the image would cease to function, because the main stream of energy would be diverted to another and simpler disposition, that which subserves the appreciation of the lines drawn on the card. But in the bypnotic state, owing to the relative dissociation and quiescence of all parts of the brain, save the disposition concerned in the production of the image, and owing to the abnormal preponderance of the excitation of this disposition over all others, the sensory impulses initiated in tho retina by the lines on the card and passing up to the brain are diverted from the channels they would normally follow and are led into this preponderantly excited disposition. This disposition thereupon receives its stimulus, not only from other parts of the cortex in the way characteristic of ideational process, but directly from the sensory nerve tract; and this direct reception of sensory impulses seems to be in all cases the condition of the perceptual character or sensory vividness of a presentation. This view of the process of induction of the hypnotic hallucination brings it into line with the " recurrent sonse-impressions" which are apt to follow on prolonged work witb the microscope on some one kind of structure, or on other constantly repeated sense-impressions; and also with the patbological hallucinations that seem to arise from morbid irritability of some brain tract. For in all such cases the hallucination may be plausibly regarded as due to the diversion of sensory impulses from their normal channels and their attraction into the over-excited system or disposition. 
in normal fashion, and is apparently not conscious of it, his neglect and active avoidance of it show that the object is in some sense recognized ; ${ }^{1}$ and when a post-hypnotic suggestion is executed after a given number of repetitions of some signal, the signals clearly have been in some sense counted; and yet the subject remains unconscious of them. It is the facts of this order that have led so many authors to postulate a co-consciousness, a secondary stream of conscionsness split off from the primary consciousness and flowing independently of it; for the processes involved seem to be distinctly mental processes, such as normally involve consciousness. There is much to be said for that view, and also there are difficulties in the way of its acceptance. But, though we may leave the reality of such co-consciousness an open question, we are compelled by the facts of this order to believe that a complex and orderly sequence of nervous processes, dissociated from the main stream of brain activity, is involved in the execution of such tasks; the dissociation seems to circumscribe the independently operating systems.

Anresthesia also affords good evidence of dissociation; the sensory areas concerned with the reception of afferent impulses from the anasthetic part seem to be profoundly dissociated from the rest of the brain; though whether this neural dissociation suffices in itself to account for the anæsthesia, and whether we are justified in assuming, as Janet does, that the sensations of the anæsthetic limb exist, or occur, as isolated sensations or sensations of a slender stream of secondary consciousnessthese are very obscure questions which also we may leave open, while we accept the anæsthesia as positive evidence that at some point in the sensory path from the anæsthetic organ, probably a point within the cortex of the brain, resistance is abnormally increased in the way which constitutes dissociation.

The discontinuity of the memory trains of the hypnotic and the waking states, which so commonly obtains, is another piece of direct evidence of the reality of dissociation; although here, as in the preceding cases, it remains a very obscure problem: how can verbal suggestion determine the position of the line of cleavage or dissociation that separates the two systems?

A few words may be ventured as to the bearing of the views set forth above on the therapeutic applications of hypnotic suggestion. If these views represent an approximation towards the truth, it follows that the therapeutic value of hypnotic suggestion consists principally in the fact that it is a means of concentrating powerful currents of nervous

' The subject usually avoids turning bis gaze directly upou such a forbiddeu object. 
energy in any required direction and of withdrawing them from other parts. By thus withdrawing the nervous currents from an overworked or unduly irritable nervous centre or bodily organ, and by isolating it through induction of a relative dissociation of the centre, rest may be secured and a bad habit of over-action may be suspended, as, e.g., in neuralgia; while, by repeatedly directing a powerful stream of innervation through some other channel, a too sluggish organ (e.g., the bowel) nay be brought back to a more active and healthy functioning, a habit that has become disordered or unduly weakened may be restored, or a new habit may be set up to supplant, counteract, or suppress some undesirable habit. Under these two heads, the increase or the diminution of the metabolism and functioning of organs, most of the therapeutic effects of hypnotic suggestion may, I think, be classified.

There are a number of phenomena that remain very obscure, and it is not claimed that the theory of cerebral dissociation as here presented provides a complete explanation of any of the facts. But that cerebral dissociation of some degree is at least one of the essential features of the hypnotic state can, I think, hardly be doubted; and though it may be questioned by some whether even a complete account of the cerebral changes would afford anything like a complete explanation of the facts, it can hardly be disputed that any complete theory of hypnosis must take the cerebral changes into account. I venture to think that the foregoing hypothetical description of the state of the brain during hypnosis may render the conceptions of cerebral dissociation, of the peculiarity of the hypnotic dissociation, and of the process of its induction, a little clearer and more definite than they have hitherto been. It is but just to point out that the hypothesis of cerebral dissociation which I have endeavoured to develop seems to have been first suggested by Hughes Bennett, ${ }^{1}$ and that therefore, if it should ever attain to the rank of a generally accepted theory, the credit of having first enunciated it must be assigned to hin.

\section{References.}

[1] Clapanìde, E. "Théorio du sommeil," Arch. de Psych., 1906.

[2] Drssorr, Max. “Das Doppel-Ich," Leipzig, 1896.

[3] FoneL, A. “Der Hypnotismus," Stuttgart, 1902, S. 118.

[4] JANET, P. “L'automatisme psychologique," Paris, 1903.

I I am indebted to Dr. Milne Bramwell for having drawn my attention to this fact on the occasion when the substance of this paper was read to the Medical Society for the Study of Suggestive Therapeutics over which he presides. It would seem that the theory was first implied by Bennett in a lecture entitled "The Mesmeric Mania of 1851," delivered and published at Edinburgh in the year 1851. I bave not been able to refer to a copy of this lecturo. 
[5] Lipps, Тн. "Zur Psychologie der Suggestion," Leipzig, 1895, a reprint from Zcitsch. f. Hypnotismas.

[6] McDougatr, W. "On the Seat of the Psycho-physical Processes," Brain. vol. xxiv.; and "The Nature of Inhibitory Processes within the Nervous System," Brain, vol. xxvi.

[7] Ibid. "Physiological Factors of the Attention-Process," Mind, N.S. No. 47.

[8] Mũller and Pilzecker. "Experimentelle Beitrüge zur Lehre vom Gedachtniss," Zeitsch. f. Psych., Ergänzuugsbd. i., 1900.

[9] Sidis, Boris. "Psychology of Suggestion," New York, 1898.

[10] Strǜmell. Deut. Archiv. f. klin. Med., Bd. xxii.

[11] Vogt, O. "Zur. Kenntniss des Wesens und der psychologischen Bedeutung des Hypnotismus," Zeitsch. f. Hypnotismus, 1895-6. 